Palavras chave:

Barbatimão

Cerrado

Macronutrientes

Índice SPAD

Histórico:

Recebido 05/12/201 I

Aceito 25/1 I/2013

Keywords:

Barbatimão

Savannah

Macronutrients

SPAD index

Correspondência: natalia_hilgert@hotmail.com
Natália Hilgert de Souza', Marlene Estevão Marchetti², Thiago de Oliveira Carnevali², Diovany Doffinger Ramos², Silvana de Paula Quintão Scalon ${ }^{2}$, Maiby Teodoro de Oliveira ${ }^{3}$

\section{CRESCIMENTO INICIAL DE Stryphnodendron polyphylum (Mart.) EM RESPOSTA À ADUBAÇÃO COM N E P}

RESUMO: O barbatimão é uma importante espécie nativa do Cerrado, com potencial econômico e de restauração de áreas degradadas, no entanto, são escassos estudos agronômicos com a espécie, principalmente no que se refere à adubação com nitrogênio e fósforo em solos de Cerrado. Assim, objetivou-se estudar o crescimento inicial e índice SPAD em função de doses de $\mathrm{N}$ e $\mathrm{P}$ em Latossolo Vermelho distroférrico. $\mathrm{O}$ trabalho foi desenvolvido em vasos de $8 \mathrm{dm}^{3}$ e mantidos em casa de vegetação. Foram estudadas quatro doses de $\mathrm{N}\left(0 ; 20,82 ; 4 \mathrm{I}, 64\right.$ e $62,46 \mathrm{mg} \cdot \mathrm{kg}^{-1}$ de $\left.\mathrm{N}\right)$ e quatro doses de $\mathrm{P}(0 ; 4 \mathrm{I}, 72$; 83,44 e $125,16 \mathrm{mg} \cdot \mathrm{kg}^{-1}$ de $\mathrm{P}_{2} \mathrm{O}_{5}$ ), em esquema fatorial $4 \times 4$, no delineamento experimental de blocos casualizados, com quatro repetições. Decorridos 210 dias após a semeadura mediu-se a altura, o diâmetro do caule, teor indireto de clorofila das folhas, massa seca da parte aérea e radicular e teor de $\mathrm{N}$ foliar. A adubação nitrogenada e fosfatada proporcionou maior incremento para todas as características avaliadas.

\section{INITIAL GROWTH OF Stryphnodendron polyphylum (Mart.) IN RESPONSE TO FERTILIZATION WITH N AND P}

ABSTRACT: Barbatimão is an important native specie of Savannah, with economic potential and restoration of degraded areas, however, there are few agronomic studies with the specie, particularly regarding the fertilization with nitrogen and phosphorus in Cerrado soils. Thus, the objective was to study the initial growth, the levels of chlorophyll, dry matter production in function of $\mathrm{N}$ and $\mathrm{P}$ levels. The study was developed in $8 \mathrm{dm}^{3}$ pots and kept in a greenhouse. There were studied four $\mathrm{N}$ levels $(0,20.82,4 \mathrm{I} .64$ and $\left.62.46 \mathrm{mg} \mathrm{N} \mathrm{kg}^{-1}\right)$ and four $P$ levels $\left(0,41.72,83.44\right.$ and $\left.125.16 \mathrm{mg} \cdot \mathrm{kg}^{-1} \mathrm{P}_{2} \mathrm{O}_{5}\right)$ in $4 \times 4$ factorial design in randomized blocks experimental design with four replications. After 210 days after sowing height, stem diameter, chlorophyll content, dry mass of shoot and root and leaf $\mathrm{N}$ content were measured. The nitrogen and phosphate application showed higher increment for all traits. 


\section{INTRODUÇÃO}

O bioma Cerrado é considerado a savana mais rica do mundo floristicamente, em razão do seu alto endemismo, sendo uma das áreas brasileiras prioritárias para a conservação (MYERS et al., 2000). Grande parte das espécies vegetais é útil ao homem e importante para o desenvolvimento da região (BORGES FILHO; FELFILI, 2003). Entre estas, encontra-se o barbatimão (Stryphnodendron polyphyllum Mart., LeguminosaeMimosoidae), uma árvore de pequeno porte, com madeira moderadamente pesada, empregada em confecções de móveis, compensados, esculturas, lenha e carvão (LORENZI, 2002). A espécie é recomendada para a composição de reflorestamentos mistos, visando à recuperação de áreas degradadas (SOUZA; LORENZI, 2005), por ser uma espécie adaptada às condições edáficas álicas dos solos de Cerrado e por ser uma leguminosa arbórea e micorrízica (SCABORA et al., 20II).

As espécies do gênero Stryphnodendron destacam-se por suas propriedades medicinais, em razão da elevada concentração de taninos em sua casca. A casca do S. polyphyllum é utilizada popularmente como antinflamatório, antibacteriano, antidiarréico (RODRIGUES; CARVALHO, 200I), cicatrizante de lesões gástricas e contra leishmaniose (LORENZI, 2002). Herzog-Soares et al. (2006) testaram a ação do extrato bruto etanólico da casca do $S$. polyphyllum sobre a parasitemia de camundongos por Trypanosoma cruzi, e verificaram significativa redução do número de parasitos no sangue dos camundongos, podendo, dessa forma, ser alternativa no controle de parasitoses e vetores. De acordo com Borges Filho e Felfili (2003), o extrativismo da casca de barbatimão é tão forte que ocorre até mesmo em unidades de conservação, de forma desordenada, o que coloca a espécie sob risco de extinção.

O conhecimento dos aspectos nutricionais de espécies nativas do Cerrado é uma ferramenta importante para compreender $\circ$ estabelecimento destas em solos com baixa disponibilidade nutricional. Os solos sob Cerrado são ácidos, com alta saturação por alumínio e reduzida disponibilidade de nutrientes. Os teores de matéria orgânica na camada superficial variam entre 10 e $30 \mathrm{~g} \cdot \mathrm{kg}^{-1}$, resultando em baixo conteúdo de nitrogênio. O fósforo é fortemente adsorvido por óxidos de ferro e alumínio na fração argila e em argilominerais como a caulinita (PEREIRA et al., 2009). Desse modo, o nitrogênio $(N)$ e o fósforo $(P)$ são os nutrientes mais limitantes para o crescimento e desenvolvimento das plantas.
A maioria dos trabalhos com S. polyphyllum é referente a estudos farmacológicos (HERZOG-SOARES et al., 2006; LOPES et al., 2003, 2005; VINAUD et al., 2008), sendo escassos os trabalhos envolvendo o uso de fertilizantes para produção de mudas. Calgaro et al. (2008), ao estudarem os efeitos da adubação química e orgânica na fertilidade de um subsolo degradado e na micorrização do S. polyphyllum, verificaram maior crescimento das plantas, principalmente as que receberam calagem associada a $\mathrm{N}+\mathrm{P}$ e resíduos orgânicos.

Considerando-se a escassez de estudos agronômicos com o barbatimão, principalmente no que se refere à adubação em solos de Cerrado, objetivouse estudar os efeitos de doses de $\mathrm{N}$ e $\mathrm{P}$ sobre as características de crescimento e índice SPAD de mudas de barbatimão em Latossolo Vermelho distroférrico.

\section{MATERIAL E MÉTODOS}

O experimento foi desenvolvido de fevereiro a setembro de 2009, em casa de vegetação, na Universidade Federal da Grande Dourados (UFGD) em

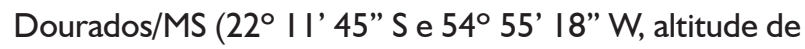
$446 \mathrm{~m})$. O clima é do tipo Cwa, segundo a classificação de Köppen, com precipitação média anual de $1500 \mathrm{~mm}$ e a temperatura média de $22^{\circ} \mathrm{C}$.

Utilizou-se um Latossolo Vermelho distroférrico de textura argilosa (EMPRESA BRASILEIRA DE PESQUISA AGROPECUÁRIA - EMBRAPA, 2006) coletado do horizonte $B$. Sua análise química foi realizada, seguindo a metodologia proposta por Claessen (1997), com o seguinte resultado: $\mathrm{pH}$ em água $=4,7 ; \mathrm{pH}$ em $\mathrm{CaCl}_{2}=4,2$; matéria orgânica $=8,2 \mathrm{~g} \cdot \mathrm{kg}^{-1} ; \mathrm{P}=1,0$ $\mathrm{mg} \cdot \mathrm{dm}^{-3} ; \mathrm{K}=0,5 \mathrm{mmol}_{\mathrm{c}} \cdot \mathrm{dm}^{-3} ; \mathrm{Ca}=4,0 \mathrm{mmol} \cdot \mathrm{dm}^{-3} ; \mathrm{Mg}$ $=2,0 \mathrm{mmol}_{\mathrm{c}} \cdot \mathrm{dm}^{-3} ; \mathrm{Al}=\mathrm{I} 4, \mathrm{I} \mathrm{mmol} \cdot \mathrm{dm}^{-3} ; \mathrm{H}+\mathrm{Al}=76,0$ $\mathrm{mmol}_{c} \cdot \mathrm{dm}^{-3} ; \mathrm{SB}=6,5 \mathrm{mmol}_{c} \cdot \mathrm{dm}^{-3} ; \mathrm{T}=82,5 \mathrm{mmol}_{\mathrm{c}} \cdot \mathrm{dm}^{-3}$ $\mathrm{e} \vee \%=7,9$.

O solo foi peneirado em malha de $6 \mathrm{~mm}$ e transferido para vasos de $8 \mathrm{dm}^{3}$. Para elevar a saturação por bases a $60 \%$, utilizou-se calcário dolomítico com PRNT 100\%, incorporado manualmente 30 dias antes da semeadura. Cada vaso foi revestido internamente com sacos plásticos para evitar a perda de água e nutrientes pela drenagem. Durante todo o período experimental os vasos foram mantidos úmidos a $70 \%$ da capacidade de campo, por meio de pesagem a cada dois dias.

As sementes de barbatimão foram coletadas em agosto de 2008, em Chapadão do Sul/MS ( $18^{\circ}$ 79' S e $52^{\circ} 62^{\prime} \mathrm{W}$ ), e armazenadas em sacos de papel em temperatura ambiente. Em fevereiro/2009 antes da semeadura, efetuou-se tratamento de quebra de 
dormência das sementes, embebendo-as em ácido sulfúrico p.a. por 20 minutos. Foram semeadas de 4 a 5 sementes por vaso, a uma profundidade de $2 \mathrm{~cm}$ e após 30 dias da germinação, realizou-se a repicagem das mudas, deixando-se as duas mais vigorosas.

Os tratamentos foram constituídos de quatro doses de $\mathrm{N}\left(0 ; 20,82 ; 41,64\right.$ e $62,46 \mathrm{mg} \cdot \mathrm{kg}^{-1}$ de $\mathrm{N}$, correspondentes a $0,50,100$ e $\left.150 \mathrm{~kg} \cdot \mathrm{ha}^{-1}\right)$ e quatro doses de $\mathrm{P}\left(0 ; 4 \mathrm{I}, 72 ; 83,44\right.$ e $125,16 \mathrm{mg} \cdot \mathrm{kg}^{-1}$ de $\mathrm{P}_{2} \mathrm{O}_{5}$, correspondentes a 0, 100, 200 e $300 \mathrm{~kg} \cdot \mathrm{ha}^{-1}$ ), utilizando como fonte ureia e superfosfato triplo, respectivamente. Estes foram arranjados em esquema fatorial $4 \times 4$, em delineamento de blocos casualizados, com quatro repetições. A adubação complementar constitui-se de $25 \mathrm{mg} \cdot \mathrm{kg}^{-1} \mathrm{~K}_{2} \mathrm{O}$, tendo como fonte o $\mathrm{KCl}$, e $150 \mathrm{mg} \cdot \mathrm{kg}^{-1}$ do formulado comercial de micronutrientes FTE-BRI2, aplicados cinco dias antes da semeadura, juntamente com ○ fósforo. A adubação nitrogenada foi parcelada, sendo um terço 40 dias após a germinação e o restante 30 dias após a primeira adubação nitrogenada.

Aos 210 dias após a semeadura (DAS) as plantas foram coletadas e avaliadas quanto à altura $(\mathrm{cm})$, do nível do solo até a gema apical; diâmetro do caule $(\mathrm{mm})$, a $\pm 1,0 \mathrm{~cm}$ do nível do solo; índice SPAD, por meio de clorofilômetro portátil Minolta SPAD - 502, efetuandose média de quatro folíolos da porção mediana da planta; massa seca da parte aérea e da raiz; e teor de $\mathrm{N}$ e $\mathrm{P}$ na parte aérea (MALAVOLTA et al., 1997).

Os dados foram submetidos à análise de variância pelo teste $\mathrm{F}$ a $5 \%$ de probabilidade e os fatores significativos analisados por meio de análise de regressão, utilizando-se programa computacional SAEG 9.0 (RIBEIRO JÚNIOR, 200I) e ajustando-se os gráficos por meio do programa Sigma Plot 9.0.

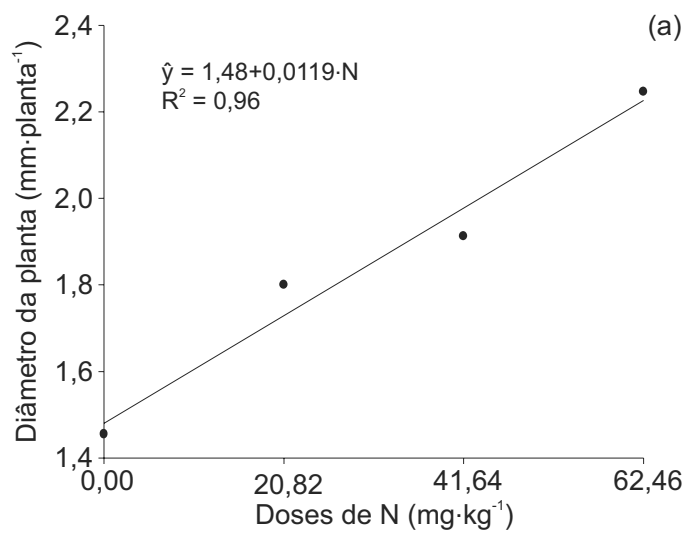

\section{RESULTADOS E DISCUSSÃO}

Para todas as características avaliadas, exceto para diâmetro do caule, houve interação significativa entre as doses de $\mathrm{N}$ e $\mathrm{P}$ utilizadas. Obteve-se altura máxima de plantas $(11,35 \mathrm{~cm}) \mathrm{com}$ as quantidades equivalentes à $55,83 \mathrm{mg}$ de $\mathrm{N} \cdot \mathrm{kg}^{-1}$ e $120,37 \mathrm{mg}$ de $\mathrm{P}_{2} \mathrm{O}_{5}$ $\mathrm{kg}^{-1}$ (Figura I). Para o diâmetro do caule os fatores isolados foram significativos, com as maiores doses de $\mathrm{N}$ e $\mathrm{P}$ proporcionando máximo de 2,22 e 2,65 mm, respectivamente (Figura 2 ).

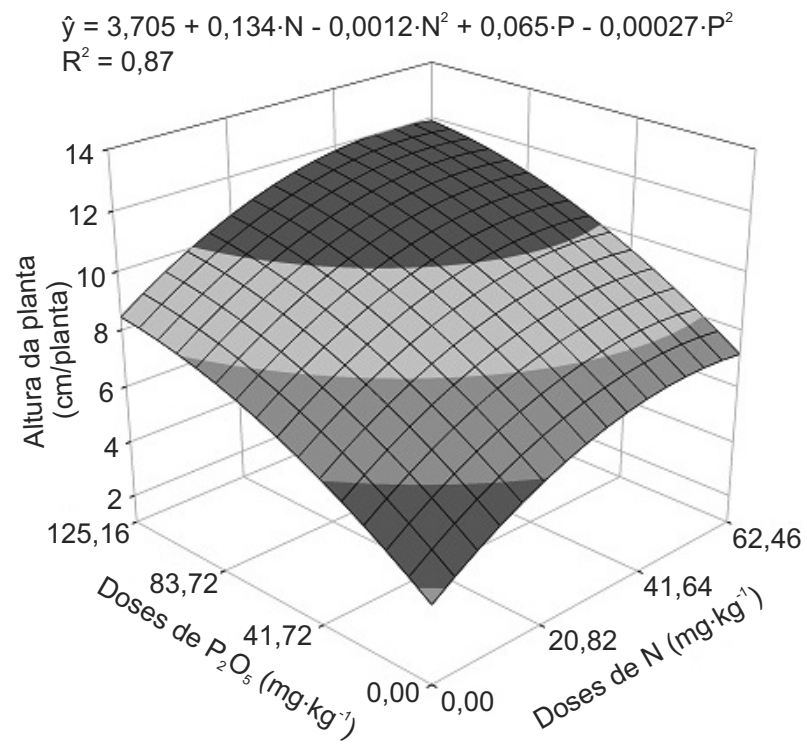

FIGURA I Altura de mudas de barbatimão aos 210 DAS, em função da adubação nitrogenada e fosfatada. C.V. $=18,5 \%$. UFGD, Dourados/MS, 2009.

FIGURE I Height of barbatimão seedlings at 210 DAS, in function of nitrogen and phosphorus fertilization. C.V. = 18.5\%. UFGD, Dourados / MS, 2009.

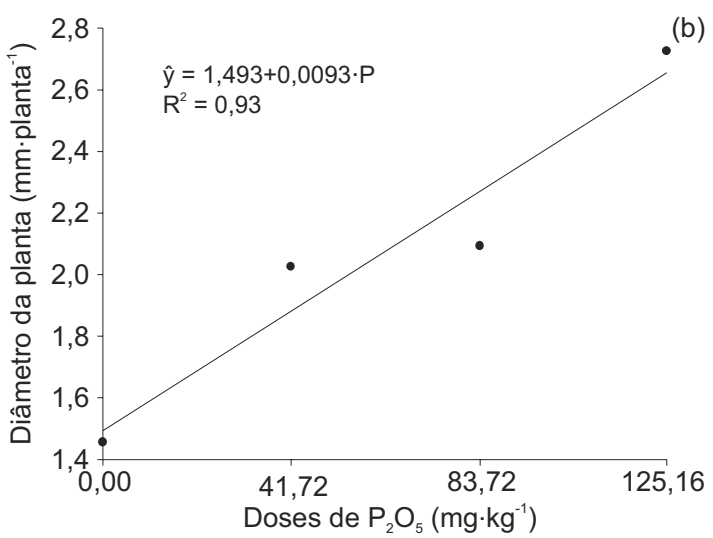

FIGURA 2 Diâmetro do caule de mudas de barbatimão aos 210 DAS, em função da adubação nitrogenada (a) e fosfatada (b). C.V. = 20,4\%. UFGD, Dourados/MS, 2009.

FIGURE 2 Stem diameter of barbatimão seedlings at 210 DAS in function of the phosphorus and nitrogen fertilization. C.V. = 20,4 \%. UFGD, Dourados/MS, 2009. 
Calgaro et al. (2008), utilizando mudas de S. polyphyllum para a recomposição de um Latossolo Vermelho distrófico degradado, em Selvíria - MS, obtiveram valores de altura e diâmetro das mudas, aos 90 dias após transplante, superiores aos observados na presente pesquisa. Nos tratamentos com adição de $\mathrm{N}+\mathrm{P}$ e calagem $+\mathrm{N}+\mathrm{P}$, os valores de altura alcançaram máximos de 18,53 e $17,63 \mathrm{~cm}$ e os de diâmetro 7,35 e 7,20 mm, respectivamente. Como fonte de $\mathrm{N}$, os autores utilizaram o sulfato de amônio na dose de $31 \mathrm{mg}$ $\mathrm{N} \mathrm{kg}^{-1}$, e $290 \mathrm{mg} \mathrm{P}_{2} \mathrm{O}_{5} \mathrm{~kg}^{-1}$ como fonte de $\mathrm{P}$, utilizando o superfosfato simples. Esses dados demonstram que o barbatimão responde positivamente à adubação.

Fernandes et al. (2008), utilizando um Latossolo Vermelho-Amarelo distrófico, avaliaram os efeitos das doses de $\mathrm{N}$ e $\mathrm{P}$ sobre o crescimento de mudas de Dimorphandra mollis Benth (faveiro), espécie pertencente à mesma subfamília (Mimosoideae). Aos 270 DAT, obtiveram valores de altura de 23,09 e 21,09 $\mathrm{cm}$ utilizando-se, respectivamente, $226 \mathrm{mg} \mathrm{N} \mathrm{dm}^{-3}$ e 390 $\mathrm{mg} \mathrm{P} \mathrm{dm}^{-3}$. Os valores máximos de diâmetro $(3,28$ e 4,25 $\mathrm{mm}$ ) foram obtidos com as doses correspondentes à 200 $\mathrm{mg} \mathrm{N} \mathrm{dm}{ }^{-3}$ e $495 \mathrm{mg} \mathrm{P} \mathrm{dm}^{-3}$, respectivamente.

Obteve-se máximo índice SPAD de 48, I (Figura 3 ), com a maior dose de $\mathrm{P}_{2} \mathrm{O}_{5}$ e dose de $\mathrm{N}$ estimada em $56,12 \mathrm{mg} \cdot \mathrm{kg}^{-1}$. O valor obtido pelo clorofilômetro portátil (índice SPAD) correlaciona-se altamente com o teor de clorofila da folha e pode identificar deficiência de $N$ (GIL et al., 2002). Essa inferência é possível pois 50 a $70 \%$ do $N$ total das folhas são integrantes de compostos associados aos cloroplastos e ao conteúdo de clorofila das folhas (CHAPMAN; BARRETO, 1997). O P desempenha importante função na transferência de energia celular, na respiração e na fotossíntese (MARSCHNER, 1997), contribuindo, assim, no aumento do teor de clorofila.

Houve correlação positiva e significativa entre o índice SPAD e teor de $\mathrm{N}$ foliar (Figura 4). Esse resultado indica a possibilidade de utilização do medidor portátil SPAD na avaliação indireta do teor de clorofila total e na caracterização do estado de $\mathrm{N}$ para o barbatimão. Pôrto et al. (20II), avaliando o emprego do índice SPAD para o diagnóstico do estado de $\mathrm{N}$ na cultura da abobrinha, também indicam a leitura SPAD para a avaliação do estado nutricional de $\mathrm{N}$, além de ser um método prático e não destrutivo. $\mathrm{O}$ teor de $\mathrm{N}$ foliar foi significativo e influenciado positivamente pela interação entre as doses $\operatorname{de~} \mathrm{N}$ e $\mathrm{P}\left(\hat{\mathrm{Y}}=18,4+0,284 \cdot \mathrm{N}-0,0021 \cdot \mathrm{N}^{2}+0,077 \cdot \mathrm{P}\right.$. $0,00088 \cdot N P ; R^{2}=0,80$ ).

O valor máximo para massa seca da parte aérea (7, I4 g) foi obtido com as maiores doses de $\mathrm{N}$ e $\mathrm{P}_{2} \mathrm{O}_{5}$, enquanto para a massa seca de raiz o maior valor $(2,32$

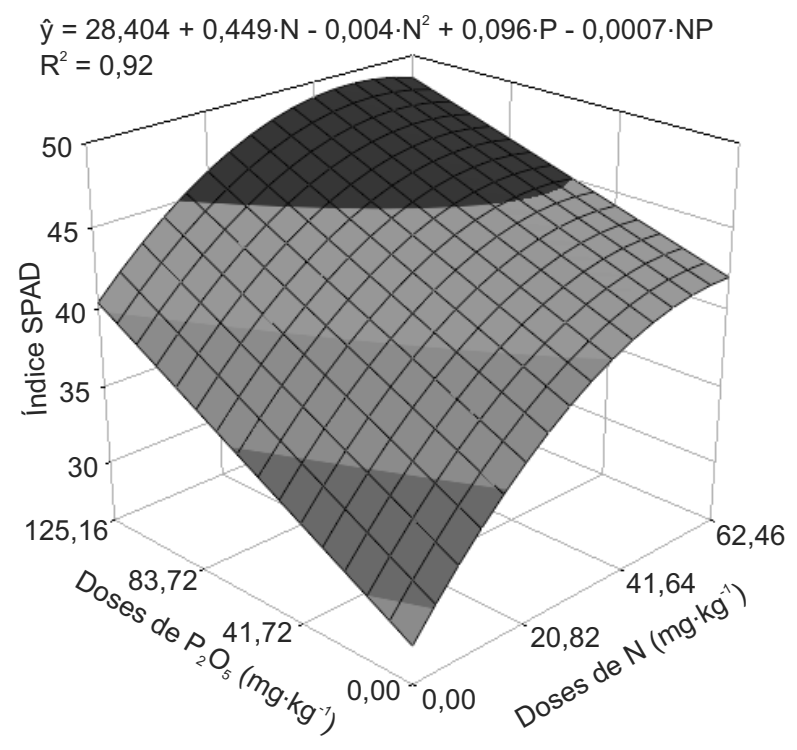

FIGURA 3 Índice SPAD em folhas de mudas de barbatimão aos 210 DAS, em função da adubação nitrogenada e fosfatada. C.V. = I3,5\%. UFGD, Dourados/ MS, 2009.

FIGURE 3 SPAD index in leaves of barbatimão seedlings at 210 DAS, in function of nitrogen and phosphorus fertilization. C.V. = 13,5\%. UFGD, Dourados/ MS, 2009.

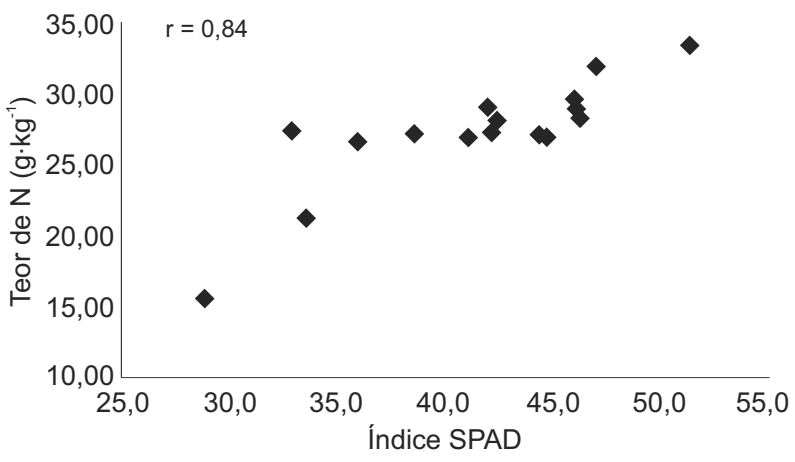

FIGURA 4 Correlação de Pearson entre teor de $\mathrm{N}$ foliar e índice SPAD em mudas de barbatimão aos 210 DAS. UFGD, Dourados/MS, 2009.

Pearson's Correlation between leaf $\mathrm{N}$ content and

FIGURE 4 SPAD index in barbatimão seedlings at 210 DAS. UFGD, Dourados/MS, 2009.

g) foi alcançado somente com a maior dose de $\mathrm{P}_{2} \mathrm{O}_{5}$ (Figura 5). É comum encontrar maior relação massa seca da parte aérea/massa seca de raiz, em fase de mudas. A primeira fase de desenvolvimento é aquela em que a plântula direciona a maior parte de suas energias para a expansão da área foliar e a formação de raízes (DEL QUIQUI et al., 2004). Na segunda fase, a partir dos 40 dias após a germinação, a demanda de nutrientes é mais intensa, em função do rápido crescimento das mudas (BARROS et al., 1997), explicando essa maior relação. 
(a) $\hat{y}=0,949+0,031 \cdot \mathrm{N}+0,009 \cdot \mathrm{P}+0,0002 \cdot \mathrm{P}^{2}$

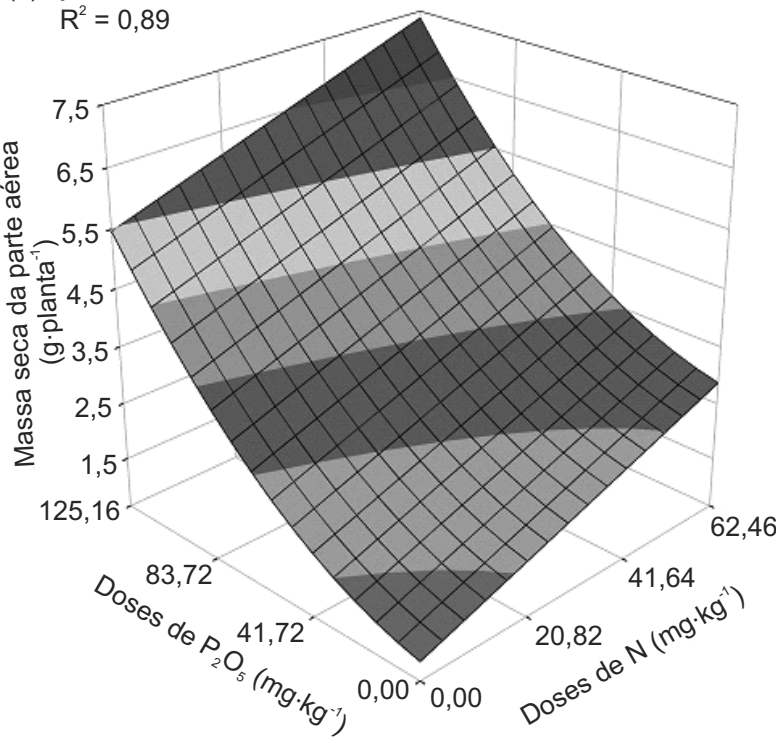

(b) $\hat{y}=0,561+0,012 \cdot \mathrm{N}+0,0053 \cdot P+0,00007 \cdot P^{2}-0,0002 \cdot N P$

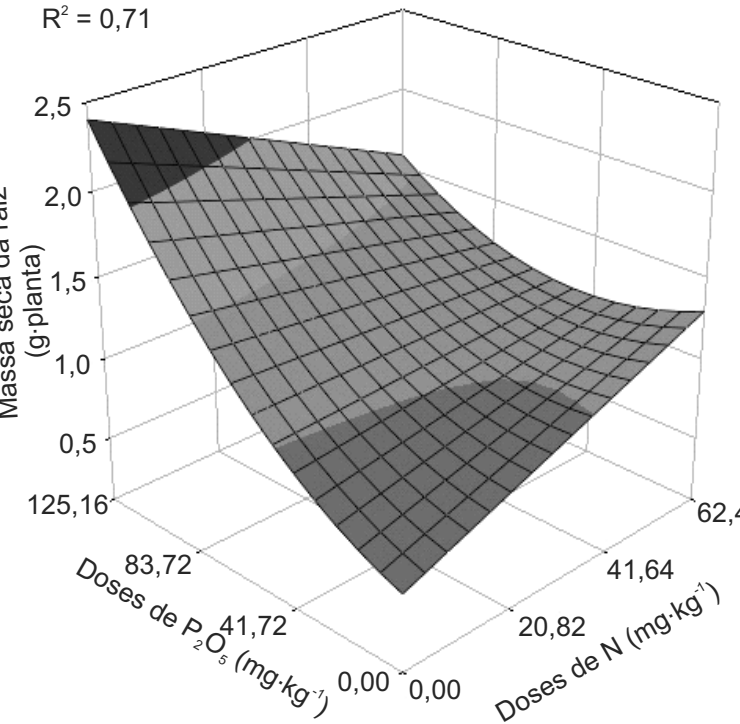

FIGURA 5 Massa seca da parte aérea (a) e da raiz (b) em mudas de barbatimão aos 210 DAS, em função da adubação nitrogenada e fosfatada. C.V. $=26,7$ e $34,9 \%$, respectivamente. UFGD, Dourados/MS 2009.

FIGURE 5 Shoot (a) and root (b) dry mass in barbatimão seedlings at 210 DAS, in function of nitrogen and phosphorus fertilization. C.V. $=26,7$ e $34,9 \%$, respectively. UFGD, Dourados/MS, 2009.

Marques et al. (2006), avaliaram o efeito de fontes e doses de $\mathrm{N}$ no crescimento inicial de Dalbergia nigra (Vell.) Fr. All. ex Benth (jacarandá-da-bahia) em diferentes tipos de solo e observaram maior aquisição de massa seca da parte aérea, aos I25 DAS, na dose de $180 \mathrm{mg} \cdot \mathrm{dm}^{-3} \mathrm{de}$ $\mathrm{N}$, tendo como fonte o sulfato de amônio em Argissolo
Vermelho-Amarelo. Venturin et al. (1999), verificaram que quando da omissão de $\mathrm{N}$ e $\mathrm{P}$ mudas de Peltophorum dubium (Sprengel) Taubert (canafístula) apresentaram crescimento muito reduzido. No tratamento completo, os autores obtiveram massa seca da parte aérea de 16,08 $\mathrm{g}$ e de raiz $8,53 \mathrm{~g}$. Esses dados corroboram com a presente pesquisa, indicando que espécies de Cerrado, em fase de muda, apresentam elevada exigência nutricional, principalmente em relação a $\mathrm{N}$ e $\mathrm{P}$.

De modo geral, o $\mathrm{P}$ foi o principal nutriente em proporcionar acúmulo de biomassa, traduzindo sua importância nos estádios iniciais de crescimento da espécie. No entanto, seu requerimento pela planta é baixo, apresentando teor de $1,64 \mathrm{mg} \cdot \mathrm{kg}^{-1}$ na parte aérea $\left(\hat{Y}=0,5 \mathrm{I} 5+0,0048 \cdot N+0,0066 \cdot P ; R^{2}=0,87\right)$, o que indica que a espécie é adaptada às condições de baixa fertilidade do solo.

Por serem os nutrientes mais limitantes para ○ desenvolvimento vegetal, $\circ \mathrm{N}$ e $\circ \mathrm{P}$ são elementos primordiais em estudos de nutrição de plantas, principalmente as de Cerrado, que carecem de informações nutricionais. Segundo Haridasan (2000), muitas espécies arbóreas nativas desenvolveram mecanismos de adaptação às condições de baixa fertilidade do solo, no entanto, essa questão deve ser comprovada por meio da capacidade de cada espécie de responder a maior disponibilidade de nutrientes. No caso do barbatimão, os maiores níveis de adubação nitrogenada e fosfatada foram responsáveis por incrementos significativos no crescimento e no valor SPAD, correlacionando-se, positivamente, com o teor de nitrogênio foliar. Esses resultados são importantes para o manejo da adubação para essa espécie, visto que são escassas informações agronômicas. Dessa forma, sugere-se que novas pesquisas sejam realizadas, no âmbito nutricional, a fim de se obter maior conhecimento quanto ao comportamento dessa espécie quanto à doses e formas de aplicação de nutrientes, não somente $\mathrm{N}$ e $\mathrm{P}$, mas de outros nutrientes.

\section{CONCLUSÕES}

De modo geral, por meio dos resultados obtidos, pode-se concluir que: a combinação das maiores dose de $\mathrm{N}$ e $\mathrm{P}$ proporcionaram maior valor do índice SPAD e maior crescimento de mudas de barbatimão, no entanto, o P mostrou-se o elemento mais importante; e o índice SPAD pode ser utilizado na avaliação indireta do teor de clorofila total e na caracterização do estado de $\mathrm{N}$ para o barbatimão. 


\section{AGRADECIMENTOS}

À Fundação de Apoio ao Desenvolvimento do Ensino, Ciência e Tecnologia do Estado de Mato Grosso do Sul (FUNDECT), pela concessão de bolsa de estudos e apoio financeiro.

\section{REFERÊNCIAS}

BARROS, N. F.; NEVES, J. C.; NOVAIS, R. F. Nutrição e adubação de eucalipto. Informe Agropecuário, Belo Horizonte, v. 18, n. 186, p. 70-75, 1997.

BORGES FILHO, H. C.; FELFILI, J. M. Avaliação dos níveis de extrativismo da casca de barbatimão [Stryphnodendron adstringens (Mart.) Coville] no Distrito Federal, Brasil. Revista Árvore, Viçosa, v. 27, n. 5, p. 735-745, 2003.

CALGARO, H. F.; VALÉRIO FILHO, W. V.; AQUINO, S. S.; MALTONI, K. L.; CASSIOLATO, A. M. R. Adubação química e orgânica na recuperação da fertilidade de subsolo degradado e na micorrização do Stryphnodendron polyphyllum. Revista Brasileira de Ciência do Solo, Viçosa, v. 32, p. I337-I347, 2008.

CHAPMAN, S. C.; BARRETO, H. J. Using a chlorophyll meter to estimate specific leaf nitrogen of tropical maize during vegetative growth. Agronomy Journal, Madison, v. 89, n. I, p. 557-562, 1997.

CLAESSEN, M. E. C. (Org.). Manual de métodos de análise de solo. 2. ed. Rio de Janeiro: EMBRAPA-CNPS, 1997. 212 p. (Documentos, I).

DELQUIQUI, E. M.; MARTINS, S. S.; PINTRO, J. C.; ANDRADE, P. J. P.; MUNIZ, A. S. Crescimento e composição mineral de mudas de eucalipto cultivadas sob condições de diferentes fontes de fertilizantes. Acta Scientiarum. Agronomy, Maringá, v. 26, n. 3, p. 293-299, 2004.

EMPRESA BRASILEIRA DE PESQUISA AGROPECUÁRIA. Centro Nacional de Pesquisa de Solos. Sistema brasileiro de classificação de solos. 2. ed. Rio de Janeiro, 2006. 306 p.

FERNANDES, L. A.; ALVES, D. S.; SILVA, L. F.; SILVA, N. C. A.; MARTINS, E. R.; SAMPAIO, R. A.; COSTA, C. A. Níveis de nitrogênio, fósforo e potássio para a produção de mudas de fava d'anta (Dimorphandra mollis Benth). Revista Brasileira de Plantas Medicinais, Botucatu, v. I0, n. I, p. 94-99, 2008.

GIL, P. T.; FONTES, P. C. R.; CECON, P. R.; FERREIRA, F. A. Índice SPAD para o diagnóstico do estado de nitrogênio e para o prognóstico da produtividade de batata. Horticultura Brasileira, Brasília, v. 20, p. 6| I-6|5, 2002.

HARIDASAN, M. Nutrição mineral de plantas nativas do cerrado. Revista Brasileira de Fisiologia Vegetal, Campinas, v. 12, p. 54-64, 2000.
HERZOG-SOARES, J. D. A.; ISAC, E.; CASTRO, A. M.; BEZERRA, J. C. B. Bioatividade de Stryphnodendron adstringens, S. Polyphyllum, Caryocar brasiliense, plantas do Cerrado brasileiro, sobre Trypanosoma cruzi "in vivo". Bioscience Journal, Uberlândia, v. 22, n. 3, p. I|3-II8, 2006.

LOPES, G. C.; NAKAMURA, C. V.; DIAS FILHO, B. P.; MELLO, J. C. P. Estudo físico-químico, químico e biológico de extrato das cascas de Stryphnodendron polyphyllum Mart. (Leguminosae). Revista Brasileira de Farmacognosia, São Paulo, v. 13, p. 24-27, 2003.

LOPES, G. C.; SANCHES, A. C. C.; NAKAMURA, C. V.; DIAS FILHO, B. P.; HEMANDES, L.; MELLO, J. C. P. Influence of extracts of Stryphnodendron polyphyllum Mart. and Stryphnodendron obovatum Benth. on the cicatrisation of cutaneous wounds in rats. Journal of Ethnopharmacology, Lausanne, v. 99, n. 2, p. 265-272, 2005.

LORENZI, H. Árvores brasileiras. 4. ed. Nova Odessa: Instituto Plantarum, 2002. v. 2, 202 p.

MALAVOlTA, E.; VITTI, G. C.; OLIVEIRA, S. A. Avaliação do estado nutricional das plantas: princípios e aplicações. Piracicaba: Associação Brasileira para a Pesquisa da Potassa e do Fosfato, 1997. 319 p.

MARQUES, V. B.; PAIVA, H. N.; GOMES, J. M.; NEVES, J. C. L.; BERNARDINO, D. C. S. Efeito de fontes e doses de nitrogênio sobre o crescimento inicial e qualidade de mudas de jacarandá-da-bahia (Dalbergia nigra (Vell.) Fr. All. ex Benth.). Revista Árvore, Viçosa, v. 30, n. 5, p. 725-735, 2006.

MARSCHNER, H. Mineral nutrition of higher plants. $2^{\text {nd }}$ ed. San Diego: Academic, 1997. 889 p.

MYERS, N.; MITTERMEIER, R. A.; MITTERMEIER, C. G.; FONSECA, G. A. B.; KENT, J. Biodiversity hotspots for conservation priorities. Nature, Oxford, v. 403, p. 853858, 2000.

PEREIRA, A. L.; CAMPOS, M. C. C.; SOUZA, Z. M.; CAVALCANTE, I. H. L.; SILVA, V. A.; MARTINS FILHO, M. V. Atributos do solo sob pastagem em sistema de sequeiro e irrigado. Ciência e Agrotecnologia, Lavras, v. 33, n. 2, p. 377-384, mar./abr. 2009.

PÔRTO, M. L.; PUIATTI, M.; FONTES, P. C. R.; CECON, P. R.; ALVES, J. C.; ARRUDA, J. A. Índice SPAD para o diagnóstico do estado de nitrogênio na cultura da abobrinha. Horticultura Brasileira, Brasília, v. 29, p. 3II-3I5, 20 I I.

RIBEIRO JÚNIOR, J. I. Análise estatística no SAEG. Viçosa, MG: UFV, 200I. 30 I p.

RODRIGUES, V. E. G.; CARVALHO, D. A. Plantas medicinais no domicílio do Cerrado. Lavras: UFLA, 200 I. 236 p. 
SCABORA, M. H.; MALTONI, K. L.; CASSIOLATO, A. M. R. Associação micorízica em espécies arbóreas, atividade microbiana e fertilidade do solo em áreas degradadas de Cerrado. Ciência Florestal, Santa Maria, v. 2I, n. 2, p. 289-30I, 20II.

SOUZA, V. C.; LORENZI, H. Botânica sistemática: guia ilustrado para identificação das famílias de Angiospermas da flora brasileira, baseado em APG II. Nova Odessa: Instituto Plantarum, 2005. 640 p.
VENTURIN, N.; DUBOC, E.; VALE, F. R.; DAVIDE, A. C. Adubação mineral do angico-amarelo (Peltophorum dubium (Spreng.) Taub.). Pesquisa Agropecuária Brasileira, Brasília, v. 34, n. 3, p. 44I-448, mar. 1999.

VINAUD, M. C.; LINO JUNIOR, R. S.; BEZERRA, J. C. B. Activity of Stryphnodendron polyphyllum, a plant from the brazilian savanah, against hemocytes of Biomphalaria glabrata, an intermediate host of Schistosoma mansoni. Revista de Patologia Tropical, Goiânia, v. 37, n. 3, p. 237-246, 2008. 
